\title{
Selection rules for Shubnikov space groups
}

\author{
P M van den Broek \\ Institute for theoretical physics, University of Nijmegan, Toernooiveld, Nijmegen, the \\ Netherlands
}

Received 17 March 1978, in final form 15 May 1978

\begin{abstract}
The theory of induced PUA representations and selection rules developed in a previous paper is applied to the Shubnikov space groups. In particular we derive for each type of Shubnikov space group an expression which governs the vanishing of matrix elements of irreducible tensor operators.
\end{abstract}

\section{Introduction}

As is well known from the results of Bargmann (1954) and Wigner (1939) the Hilbert space $\mathscr{H}$ of state vectors of a physical system with a symmetry group $G$ carries a projective unitary-antiunitary (PUA) representation $T$ of $G$. All relevant physical information is contained in matrix elements $\left(\phi_{m}^{o, a}, O_{k}^{\mu} \phi_{l}^{\nu, b}\right)$. Here the vectors $\phi_{m}^{\rho, a}$ form a basis for $\mathscr{H}$ and transform under $T$ as

$$
T(g) \phi_{m}^{\rho . a}=\sum_{n} D_{n m}^{\rho}(g) \phi_{n}^{\rho . a} \quad \forall g \in G
$$

where $D^{\rho}$ is an irreducible pUA representation of $G$ with the same factor system as $T$. The index $a$ denotes that there may be more than one subset of basis functions transforming according to $D^{\rho}$. Further $O^{\mu}$ is an irreducible tensor operator transforming according to the irreducible UA representation $D^{\mu}$ of $G$ :

$$
T(g) O_{k}^{\mu} T(g)^{-1}=\sum_{n} D_{n k}^{\mu}(g) O_{n}^{\mu} \quad \forall g \in G .
$$

In a previous paper (van den Broek 1978, hereafter referred to as I) we derived selection rules for these matrix elements, especially for the case where the irreducible PUA representations of $G$ are obtained with the procedure of generalised induction given by Shaw and Lever (1974). In this paper we will apply the results of I to the Shubnikov space groups. For the Shubnikov space groups of type I these selection rules have already been obtained for the case of vector representations (Bradley 1966, Cracknell and Davies 1976, $\$ 4.7$ of Bradley and Cracknell 1972). The definitions of a factor system, of a PUA representation and of an induced PUA representation have been given in I and will therefore not be repeated here.

Since Shubnikov space groups are infinite groups one usually applies periodic boundary conditions to make the groups finite and the irreducible PUA representations finite-dimensional. Here we will not apply period boundary conditions, but consider only finite-dimensional irreducible PUA representations. 


\section{Selection rules for Shubnikov space groups of types I and III}

Let $G$ be a Shubnikov space group of type I or III, $H$ its subgroup of translations and $G_{0}$ its non-magnetic subgroup. The Shubnikov point groups $K$ and $K_{0}$ are defined by $G / H$ and $G_{0} / H$ respectively. The identities of $H$ and $K$ will be denoted by $e$ and $E$ respectively. Elements of $G$ will be denoted by $(t, R)$ where $t \in H$ and $R \in K$. We define $(t, R)$ by its action on space-time:

$$
(t, R)(x ; t)=\left(R x+t+t_{R} ; \epsilon_{R} t\right)
$$

where $\epsilon_{R}$ is defined by

$$
\epsilon_{R}=\left\{\begin{aligned}
1 & \text { if } R \in K_{0} \\
-1 & \text { if } R \notin K_{0}
\end{aligned}\right.
$$

and $t_{R}$ is a fixed non-primitive translation associated with $R$. The multiplication of elements of $G$ is given by

$$
(t, R)\left(t^{\prime}, R^{\prime}\right)=\left(t+R t^{\prime}+m\left(R, R^{\prime}\right), R R^{\prime}\right)
$$

where the mapping $\boldsymbol{m}: K \times K \rightarrow H$ is given by

$$
m\left(R, R^{\prime}\right)=t_{R}+R t_{R^{\prime}}=t_{R R^{\prime}}
$$

Let $t_{1}, t_{2}$ and $t_{3}$ be basic translations of $H$. Then each element $t$ of $H$ can be written as $t=n_{1} t_{1}+n_{2} t_{2}+n_{3} t_{3}$ for some integers $n_{1}, n_{2}$ and $n_{3}$. In the sequel we shall identify $t$ with the column vector with entries $n_{1}, n_{2}$ and $n_{3}$. Moreover each element $R$ of $K$ is given by the integer $3 \times 3$ matrix which represents $R$ with respect to the basic vectors $t_{1}, t_{2}$ and $t_{3}$, which is also denoted by $R$.

Let $\sigma$ be a factor system of $G$. From two previous papers (van den Broek $1977 \mathrm{a}, \mathrm{b}$ ) it follows that we can choose the basic translations and the factor system $\sigma$ from its equivalence class in such a way that

$$
\sigma\left((t, R)\left(t^{\prime}, R^{\prime}\right)\right)=\gamma\left(t, R t^{\prime}\right) \gamma\left(t+R t^{\prime}, \boldsymbol{m}\left(R, R^{\prime}\right)\right) \nu\left(R, R^{\prime}\right) P\left(R, t^{\prime}\right)
$$

where

$$
\gamma\left(t, t^{\prime}\right)=\exp \left[-2 \pi i\left(t^{\mathrm{T}} A \boldsymbol{t}^{\prime}\right)\right]
$$

Here $t^{\mathrm{T}}$ denotes the transpose of $t$ and $A$ is a $3 \times 3$ matrix of the form

$$
A=\left(\begin{array}{ccc}
0 & m / 2 N & 0 \\
-m / 2 N & 0 & 0 \\
0 & 0 & 0
\end{array}\right)
$$

where $m$ and $N$ are integers with no common factor.

Furthermore, for each $R \in K$ there exists a symmetric $3 \times 3$ matrix $B^{R}$ with half-integer elements and a column vector $k(R)$ such that

$$
\epsilon_{R} R^{-1 \mathrm{~T}} A R^{-1}-A=B^{R} \quad(\bmod 1)
$$

and

$$
P(R, t)=\exp \left(\pi \mathrm{i} t^{\mathrm{T}} R^{\mathrm{T}} B^{R} R t\right) \exp (-2 \pi \mathrm{i} k(R) . R t) .
$$

So the factor system $\sigma$ is given by $m, N$, the vectors $k(R)$, the matrices $B^{R}$ and the mapping $\nu: K \times K \rightarrow U(1)$. 
The irreducible PUA representations of $G$ with factor system $\sigma$ are obtained from the irreducible pu representations of $H$ with factor system $\gamma$ with the procedure of generalised induction (for a brief description of this procedure see van den Broek 1977b). The irreducible PU representations of $H$ with factor system $\gamma$ are all $N$ dimensional and labelled by a vector $k$, the three components of which run through the interval $[0,1)$. Their matrix elements are given by

$$
\left[D^{k}(t)\right]_{l l}= \begin{cases}\exp \left[2 \pi \mathrm{i}\left(\frac{k_{1}}{N} t_{1}+\frac{k_{2}}{N} t_{2}+k_{3} t_{3}+\frac{k_{2}}{N}\right)(j-i)-\frac{m}{N} t_{1}\left(\frac{1}{2} t_{2}+j\right)\right] \\ \text { if }\left(t_{2}+j-i / N\right) \text { is integer } \\ \text { otherwise. }\end{cases}
$$

For each $R \in K$ the pu representation $D_{R}^{k}$ of $H$ is defined by

$$
D_{R}^{k}(t)=\left[D^{k}\left(R^{-1} t\right)\right]^{R} P^{*}\left(R, R^{-1} t\right)
$$

where, if $M$ is a scalar or a matrix, $M^{R}$ is defined by

$$
M^{R}= \begin{cases}M & R \in K_{0} \\ M^{*} & R \notin K_{0}\end{cases}
$$

and the asterisk denotes complex conjugation.

With the criterion derived in van den Broek (1977b) one can easily check whether or not $D^{k}$ and $D_{R}^{k}$ are equivalent.

Let $K^{k}$ be the subgroup of $K$ defined by $K^{k}=\left\{R \in K \mid D_{R}^{k} \sim D^{k}\right\}$. The orbit of $D^{k}$ is defined by $\operatorname{Orb}\left(D^{k}\right)=\left\{D_{R}^{k} \mid R \in K\right\}$ and the little group $L^{k}$ of $D^{k}$ is defined by $L^{k}=\left\{(t, R) \mid R \in K^{k}\right\}$. For each $R \in K^{k}$ there exists a unitary matrix $U_{k}(R)$ with the property

$$
D^{k}(t)=U_{k}(R) D_{R}^{k}(t) U_{k}^{-1}(R) .
$$

If we define

$$
U_{k}(t, R)=D^{k}(t) U_{k}(R) \quad \forall(t, R) \in L^{k}
$$

then $U_{k}$ is a PUA representation of $L^{k}$ with a factor system $\omega$ which can be shown to be of the form (van den Broek 1976, 1977a)

$$
\omega\left((t, R)\left(t^{\prime}, R^{\prime}\right)\right)=\gamma\left(t, R t^{\prime}\right) \gamma\left(t+R t^{\prime}, \boldsymbol{m}\left(R, R^{\prime}\right)\right) \mu\left(R, R^{\prime}\right) P\left(R, t^{\prime}\right) .
$$

Thus the factor system $\left(\sigma \downarrow\left(L^{k} \times L^{k}\right)\right) \omega^{*}$ reduces to a factor system $\nu \mu^{*}$ of $K^{k}$. If $E$ is an irreducible PUA representation of $K^{k}$ with factor system $\nu \mu^{*}$, then $\Delta$, defined by

$$
\Delta(t, R)=U_{k}(t, R) \otimes E(R) \quad \forall(t, R) \in L^{k}
$$

is an irreducible PUA representation of $L^{k}$ with factor system $\sigma \downarrow\left(L^{k} \times L^{k}\right)$. A complete set of inequivalent irreducible pUA representations of $G$ with factor system $\sigma$ is given by $\left\{D \mid D=\Delta_{\sigma}^{\uparrow} G\right\}$, where $\Delta$ is given by equation (2.16); $E$ runs through a complete set of inequivalent irreducible PUA representations of $K^{k}$ with factor system $\nu \mu^{*}$ and, from each orbit of irreducible PU representations of $H$ with factor system $\gamma$, one representative is taken.

In I we deduced that the matrix elements $\left(\phi_{m}^{\rho, a}, O_{k}^{\mu} \phi_{l}^{\nu, b}\right)$ can only be non-zero if the trivial UA representation of $G$ occurs in the triple direct product $D^{\mu} \otimes D^{\rho^{*}} \otimes D^{\nu}$ (notation as in the introduction). Suppose that the irreducible PUA representations $D^{\rho}$ 
and $D^{\nu}$ of $G$ with factor system $\sigma$ and the irreducible UA representation $D^{\mu}$ of $G$ have been obtained with the procedure of generalised induction as described above:

$$
D^{\mu}=\Delta^{\mu} \uparrow G, \quad D^{\rho}=\Delta_{\sigma}^{\rho \uparrow} G, \quad D^{\nu}=\Delta \prod_{\sigma} G .
$$

$\Delta^{\mu}, \Delta^{\rho}$ and $\Delta^{\nu}$ have the form of equation (2.16) and are defined on $L^{k^{\prime \prime}}, L^{k^{\prime}}$ and $L^{k}$ respectively, which are the little groups of $D^{k^{\prime \prime}}, D^{k^{\prime}}$ and $D^{k}$ respectively. Here $D^{k}$ and $D^{k^{\prime}}$ are irreducible PU representations of $H$ with factor system $\gamma$ and are given by equation (2.10), and $D^{k^{\prime \prime}}$ is a one-dimensional representation of $H$ :

$$
D^{k^{\prime \prime}}(\boldsymbol{t})=\exp \left(2 \pi \mathrm{i} \boldsymbol{k}^{\prime \prime} \cdot t\right)
$$

Now factorise $G$ into double cosets as follows:

$$
G=\sum_{i} L^{k}\left(e, R_{1}\right) L^{k^{\prime}}
$$

and let $L_{1}^{k^{\prime}}$ be given by $L_{i}^{k^{\prime}}=\left(e, R_{i}\right) L^{k^{\prime}}\left(e, R_{i}\right)^{-1}$ and $L_{i}^{k^{\prime}, k}$ by $L_{i}^{k^{\prime}} \cap L^{k}$ for each double coset representatative $\left(e, R_{i}\right)$. Again factorise $G$ into double cosets for each $i$ as follows:

$$
G=\sum_{j} L_{i}^{\boldsymbol{k}^{\prime}, \boldsymbol{k}}\left(\boldsymbol{e}, \boldsymbol{R}_{i, i}\right) L^{\boldsymbol{k}^{\prime \prime}}
$$

and define $L_{j, t}^{k^{\prime \prime}}$ and $M$ by $L_{j, l}^{k^{\prime \prime}}=\left(e, R_{j, i}\right) L^{k^{\prime \prime}}\left(e, R_{j, 1}\right)^{-1}$ and $M=L_{j, l}^{k^{\prime \prime}} \cap L_{i}^{k^{\prime}, \boldsymbol{k}} \cap G_{0}$ respectively. Note that $M$ is a subgroup of $G_{0}$ and contains $H$ as an invariant subgroup. The PUA representation $\Delta_{i}^{\rho}$ of $L_{i}^{k^{\prime}}$ with factor system $\sigma \downarrow\left(L_{i}^{k^{\prime}} \times L_{l}^{k^{\prime}}\right)$ is defined by

$$
\begin{aligned}
& \Delta_{i}^{\rho}\left(\left(e, R_{i}\right)(t, R)\left(e, R_{1}\right)^{-1}\right) \\
&= \sigma\left(\left(e, R_{i}\right)(t, R)\left(e, R_{i}\right)^{-1},\left(e, R_{i}\right)\right) \sigma^{*}\left(\left(e, R_{i}\right),(t, R)\right)\left[\Delta^{o}(t, R)\right]^{R_{i}} \\
& \forall(t, R) \in L^{k^{\prime}}
\end{aligned}
$$

and the UA representation $\Delta_{j, t}^{\mu}$ of $L_{j, l}^{k^{\prime \prime}}$ by

$$
\Delta_{i, i}^{\mu}\left(\left(e, R_{j, i}\right)(t, R)\left(e, R_{t, i}\right)^{-1}\right)=\left[\Delta^{\mu}(t, R)\right]^{R_{1, i}} \quad \forall(t, R) \in L^{k^{\prime \prime}} .
$$

From I it follows that the trivial UA representation of $G$ occurs in the triple direct product $D^{\mu} \otimes D^{\rho^{*}} \otimes D^{\nu}$ if and only if the trivial representation of $M$ occurs in the triple direct product $\left(\Delta_{i, i}^{\mu} \downarrow M\right) \otimes\left(\Delta_{i}^{p} \downarrow M\right)^{*} \otimes\left(\Delta^{\nu} \downarrow M\right)$ for some $i$ and $j$. This is the case if and only if

$$
\sum_{(t, R) \in M} \operatorname{Tr} \Delta_{j, i}^{\mu}(t, R) \operatorname{Tr} \Delta_{i}^{\rho^{*}}(t, R) \operatorname{Tr} \Delta^{\nu}(\boldsymbol{t}, R) \neq 0
$$

for some $i$ and $j$.

For irreducible unitary vector representations the summation over the translations in the left-hand side of equation (2.20) can be carried out, giving the desired selection rules immediately (Bradley 1966, Bradley and Cracknell 1972). However, this is not possible in the general case, since $D^{k}$ and $D^{k^{\prime}}$ are $N$-dimensional instead of onedimensional. Let us therefore consider the subgroup $H_{1}$ of $H$, defined by

$$
H_{1}=\left\{t \in H \mid t_{1}=n_{1} N ; t_{2}=n_{2} N ; n_{1}, n_{2} \text { integer }\right\} .
$$

This subgroup has two important properties; it is invariant under the matrices $R$ of $K$ (van den Broek 1977b) and on $H_{1}, D^{k}$ and $D^{k^{\prime}}$ are multiples of the unit matrix

$$
D^{k}(Q t)=\left[\exp \left\{2 \pi \mathrm{i}\left(\boldsymbol{k}, t-\frac{1}{2} m N t_{1} t_{2}\right)\right\}\right] I
$$


and similarly for $\boldsymbol{k}^{\prime}$, where $\mathbf{l}$ is the $N$-dimensional unit matrix and $Q$ is given by

$$
Q=\left(\begin{array}{ccc}
N & 0 & 0 \\
0 & N & 0 \\
0 & 0 & 1
\end{array}\right)
$$

Any $t \in H$ can uniquely be written as $t=Q s+w$, where $s \in H, 0 \leqslant w_{1}, w_{2}<N$ and $w_{3}=0$. Our purpose is to write equation (2.20) for each $i$ and $j$ in the form

$$
\sum_{\boldsymbol{w}, R} f_{1}(\boldsymbol{w} ; R) \sum_{s} f_{2}(\boldsymbol{s} ; \boldsymbol{w} ; R) \neq 0
$$

and then to carry out the summation over $s$.

Therefore we will evaluate the three traces appearing in equation (2.20), put all factors not depending on $s$ in $f_{1}$ and forget about them; it will turn out that $f_{2}$ is independent of $\boldsymbol{w}$ and $R$. Of these calculations only an outline will be given. The result is given in equation (2.30). Let us first consider the term $\operatorname{Tr} \Delta_{j, i}^{\mu}(t, R)$. We first use equation (2.19), then use the fact that $\Delta^{\mu}$ is a UA representation of $L^{k^{\prime \prime}}$ to split off the term $\left[\Delta^{\mu}\left(R_{j, i}^{-1} Q s, E\right)\right]^{R_{, i}}$ and finally apply the equations (2.16) and (2.14). We then find that the only term dependent on $s$ is $\exp \left\{2 \pi \mathrm{i} \epsilon_{R_{, t}} \boldsymbol{k}^{\prime \prime}, R_{j, t}^{-1} Q s\right\}$.

Next consider the term $\operatorname{Tr} \Delta^{\nu}(t, R)$. We can split off the term $\Delta^{\nu}(Q s, E)$ since $\Delta^{\nu}$ is a PUA representation of $L^{k}$ and then use equation (2.5) to handle the factor system. Applying the equations (2.16), (2.14) and (2.10) shows that the $s$-dependent terms are $\gamma^{*}(Q s, \boldsymbol{w}) \exp \left\{\left[2 \pi \mathrm{i}\left(\boldsymbol{k} . \boldsymbol{s}-\frac{1}{2} m N s_{1} s_{2}\right)\right\}\right.$.

Finally we consider the term $\operatorname{Tr} \Delta_{i}^{\rho}(t, R)$. First we use equation (2.18) and then split off the term $\left[\Delta^{\rho^{*}}\left(R_{i}^{-1} Q s, E\right)\right]^{R_{t}}$. The factor systems are handled again with equation (2.5). If we now apply the equations (2.16), (2.14) and $(2.10)$ we find that the $s$-dependent terms are

$$
\begin{aligned}
& \gamma^{*}\left(Q s, \boldsymbol{m}\left(R, R_{i}\right)\right) \gamma\left(Q s, m\left(R_{i}, R_{i}^{-1} R R_{i}\right)\right) \\
& \gamma^{R_{i}}\left(R_{i}^{-1} Q s, R_{i}^{-1} w-m\left(R_{i}^{-1}, R_{i}\right)+R_{i}^{-1} \boldsymbol{m}\left(R, R_{i}\right)+\boldsymbol{m}\left(R_{i}^{-1}, R R_{i}\right)\right) \\
& P\left(R_{i}, R_{i}^{-1} Q s+R_{i}^{-1} w-m\left(R_{i}^{-1}, R_{i}\right)+R_{i}^{-1} \boldsymbol{m}\left(R, R_{i}\right)+\boldsymbol{m}\left(R_{i}^{-1}, R R_{i}\right)\right) \\
& \exp \left\{-2 \pi i \epsilon_{R_{i}}\left(\boldsymbol{k}^{\prime} . Q^{-1} R_{i}^{-1} Q s-\frac{1}{2} m N\left(Q^{-1} R_{i}^{-1} Q s\right)_{1}\left(Q^{-1} R_{i}^{-1} Q s\right)_{2}\right)\right\}
\end{aligned}
$$

From equation (3.4) of van den Broek (1976) it follows that

$$
\begin{aligned}
& P\left(R_{i}, R_{i}^{-1} Q s+R_{i}^{-1} w-m\left(R_{i}^{-1}, R_{i}\right)+R_{i}^{-1} \boldsymbol{m}\left(R, R_{i}\right)+m\left(R_{i}^{-1}, R R_{i}\right)\right)=P\left(R_{i}, R_{i}^{-1} Q s\right) \\
& P\left(R_{i}, R_{i}^{-1} w-m\left(R_{i}^{-1}, R_{i}\right)+R_{i}^{-1} \boldsymbol{m}\left(R, R_{i}\right)+\boldsymbol{m}\left(R_{i}^{-1}, R R_{i}\right)\right) \\
& \gamma\left(Q s, w-R_{i} \boldsymbol{m}\left(R_{i}^{-1}, R_{i}\right)+\boldsymbol{m}\left(R, R_{i}\right)+R_{i} \boldsymbol{m}\left(R_{i}^{-1}, R R_{i}\right)\right) \\
& \gamma^{* R_{i}}\left(R_{i}^{-1} Q s, R_{i}^{-1} w-m\left(R_{i}^{-1}, R_{i}\right)+R_{i}^{-1} \boldsymbol{m}\left(R, R_{i}\right)+\boldsymbol{m}\left(R_{i}^{-1}, R R_{i}\right)\right) .
\end{aligned}
$$

Now we take the $s$-dependent factors of the three terms together, substitute the right-hand side of equation (2.26) for its left-hand side, leave out the second term of this right-hand side since it does not depend on $s$ and simplify the result using the relations

$$
\begin{array}{ll}
\gamma\left(t, t^{\prime}\right)=\gamma^{*}\left(t,-t^{\prime}\right) & \forall t, t^{\prime} \in H \\
\gamma\left(t, t^{\prime}+t^{\prime \prime}\right)=\gamma\left(t, t^{\prime}\right) \gamma\left(t, t^{\prime \prime}\right) & \forall t, t^{\prime}, t^{\prime \prime} \in H
\end{array}
$$


which follow immediately from equation (2.6), and

$\boldsymbol{m}\left(R, R^{\prime}\right)+\boldsymbol{m}\left(R R^{\prime}, R^{\prime \prime}\right)=\boldsymbol{m}\left(R, R^{\prime} R^{\prime \prime}\right)+R \boldsymbol{m}\left(R^{\prime}, R^{\prime \prime}\right) \quad \forall R, R^{\prime}, R^{\prime \prime} \in K$

which follows immediately from the definition of the mapping $m: K \times K \rightarrow H$. Finally we find that $f_{2}(s ; \boldsymbol{w} ; R)$ is independent of $\boldsymbol{w}$ and $R$ and, if we use equation (2.9), is given by

$$
\begin{aligned}
f_{2}(s)=\exp [2 & \pi i\left\{\epsilon_{R_{l,}} \boldsymbol{k}^{\prime \prime} . R_{j, l}^{-1} Q s-\epsilon_{R_{t}} \boldsymbol{k}^{\prime} \cdot Q^{-1} R_{l}^{-1} Q s\right. \\
& +\frac{1}{2} n N \epsilon_{R_{t}}\left(Q^{-1} R_{i}^{-1} Q s\right)_{1}\left(Q^{-1} R_{i}^{-1} Q s\right)_{2}+\boldsymbol{k} \cdot \boldsymbol{s} \\
& \left.\left.-\frac{1}{2} m s_{s_{1}} s_{2}+\frac{1}{2} s^{\mathrm{T}} Q B^{R_{i}} Q s-k\left(R_{\imath}\right) \cdot Q s\right\}\right] .
\end{aligned}
$$

Let us first consider the function $\phi(s)$ defined by

$\phi(s)=\frac{1}{2} m N \epsilon_{R_{i}}\left(Q^{-1} R_{i}^{-1} Q s\right)_{1}\left(Q^{-1} R_{i}^{-1} Q s\right)_{2}-\frac{1}{2} m N s_{1} s_{2}+\frac{1}{2} s^{\mathrm{T}} Q B^{R_{i}} Q s$.

Then

$\phi\left(s+s^{\prime}\right)-\phi(s)-\phi\left(s^{\prime}\right)=\frac{1}{2} m N \epsilon_{R_{i}} s^{\mathrm{T}} Q R_{1}^{-1 \mathrm{~T}} Q^{-1} C Q^{-1} R_{i}^{-1} Q s^{\prime}-\frac{1}{2} m N s^{\mathrm{T}} C s^{\prime}+s^{\mathrm{T}} Q B^{R_{i}} Q s^{\prime}$

where

$$
C=\left(\begin{array}{lll}
0 & 1 & 0 \\
1 & 0 & 0 \\
0 & 0 & 0
\end{array}\right) .
$$

From equation (2.8) it follows that

$$
B^{R}=-\epsilon_{R} R^{-1 \mathrm{~T}} B^{\left(R^{-1}\right)} R^{-1} \quad(\bmod 1) .
$$

Therefore the right-hand side of equation $(2.32)$ is equal $(\bmod 1)$ up to a sign to the right-hand side of equation (4.20) of van den Broek (1977b) (if $s$ is replaced by $t$ and $R_{i}^{-1}$ by $R$ ). But this right-hand side was proved to be an integer; thus $\phi\left(s+s^{\prime}\right)-$ $\phi(s)-\phi\left(s^{\prime}\right)$ is integral and we may write

$$
\phi(s)=p\left(R_{\imath}\right) . s \quad(\bmod 1) .
$$

From equation (2.31) it follows that we can choose $p\left(R_{i}\right)$ by

$$
\left[\boldsymbol{p}\left(R_{i}\right)\right]_{k}=\frac{\epsilon_{R_{i}} m}{2 N} Q_{k k}^{2}\left(R_{i}^{-1}\right)_{1 k}\left(R_{i}^{-1}\right)_{2 k}+\frac{1}{2} Q_{k k}^{2} B_{k k}^{R} .
$$

Equation (2.30) now becomes

$$
f_{2}(s)=\exp \left\{2 \pi \mathrm{i} s .\left(\epsilon_{R_{j, i}} Q R_{j, i}^{-1 \mathrm{~T}} \boldsymbol{k}^{\prime \prime}-\epsilon_{R_{i}} Q R_{i}^{-1 \mathrm{~T}} Q^{-1} \boldsymbol{k}^{\prime}+\boldsymbol{k}-Q \boldsymbol{k}\left(R_{i}\right)+\boldsymbol{p}\left(R_{i}\right)\right)\right\} \text {. }
$$

So $\Sigma_{s} f_{2}(s)$ is equal to zero unless

$$
\epsilon_{R_{t, i}} Q R_{j, i}^{-1 \top} k^{\prime \prime}-\epsilon_{R_{i}} Q R_{,}^{-1 \top} Q^{-1} k^{\prime}+k-Q k\left(R_{i}\right)+p\left(R_{i}\right)=0 \quad(\bmod 1)
$$

and our final result is that the matrix elements $\left(\phi_{m}^{p, a}, O_{k}^{\mu} \phi_{l}^{\nu, b}\right)$ are equal to zero for each $m, k$ and $l$ unless there exist values for $i$ and $j$ such that equation (2.38) holds. Note that the results of Bradley (1966) and Bradley and Cracknell (1972) for the case of unitary representations are re-obtained by putting $\epsilon_{R_{i, t}}=\epsilon_{R_{i}}=1, k\left(R_{i}\right)=p\left(R_{i}\right)=0$ and replacing $Q$ by the unit matrix. 


\section{Selection rules for Shubnikov space groups of types II and IV}

Since the derivation of selection rules for the Shubnikov space groups of type II and type IV is in many respects analogous to the derivations of the previous section, we will not give here the complete derivation; instead we will restrict ourselves to mentioning the differences with the previous section.

Let $\boldsymbol{G}$ be a Shubnikov space group of type II and $G$ its unitary subgroup which is a type I Shubnikov space group. The notation of elements of $G$ will be as in the previous section. Elements of $G$ will be denoted by $(t, R, \epsilon)$ where $(t, R) \in G$ and $\epsilon$ equals \pm 1 . We define $(t, R, \epsilon)$ by its action on space-time:

$$
(t, R, \epsilon)(x ; t)=\left(R x+t+t_{R} ; \epsilon t\right) .
$$

The multiplication of elements of $\boldsymbol{G}$ is given by

$$
(t, R, \epsilon)\left(t^{\prime}, R^{\prime}, \epsilon^{\prime}\right)=\left(t+R t^{\prime}+m\left(R, R^{\prime}\right), R R^{\prime}, \epsilon \epsilon^{\prime}\right) .
$$

Let $\omega$ be a factor system of $\boldsymbol{G}$. From van den Broek (1977a, b) it follows that $\omega$ can be chosen from its equivalence class in such a way that

$$
\omega\left((t, R, \epsilon),\left(t^{\prime}, R^{\prime}, \epsilon^{\prime}\right)\right)=\sigma\left((t, R),\left(t^{\prime}, R\right)\right) N\left(\epsilon, \epsilon^{\prime}\right) M\left(\epsilon ;\left(t^{\prime}, R^{\prime}\right)\right)
$$

where $\sigma$ is a factor system of $G$ which has all the properties of the previous section, and $M$ has the properties

$$
M(1 ;(t, R))=1 \quad \forall(t, R) \in G
$$

and

$$
M(-1,(\boldsymbol{t}, E))=\exp \left\{2 \pi i\left[(m / N) t_{1} t_{2}+\boldsymbol{k}_{-1} \cdot \boldsymbol{t}\right]\right\}
$$

for some vector $\boldsymbol{k}_{-1}$.

For each irreducible PU representation $D^{k}$ of $H$ with factor system $\gamma$, and each coset representative $(\boldsymbol{e}, R, \epsilon)$ of $\boldsymbol{G}$ with respect to $H$, the irreducible PU representation $D_{(R, \epsilon)}^{k}$ of $H$ is defined by

$$
D_{(R, \epsilon)}^{k}(t)=\left[D^{k}\left(R^{-1} t\right)\right]_{\epsilon} P^{*}\left(R, R^{-1} t\right) M^{*}\left(\epsilon ;\left(R^{-1} t, E\right)\right)
$$

where, if $M$ is a scalar or a matrix, $M_{\epsilon}$ is defined by $M_{1}=M$ and $M_{-1}=M^{*}$.

Analogous to the derivation of equation (2.20) we obtain

$$
\sum_{(t, R, 1) \in M} \operatorname{Tr} \Delta_{j, i}^{\mu}(t, R, 1) \operatorname{Tr} \Delta_{i}^{\rho^{*}}(t, R, 1) \operatorname{Tr} \Delta^{\nu}(t, R, 1) \neq 0
$$

the only difference with the previous section being that the double coset representatives are given here by $\left(e, R_{i}, \epsilon_{i}\right)$ and $\left(e, R_{j, i}, \epsilon_{j, i}\right)$ and the upper indices $R$ are replaced by lower indices $\epsilon$. The first and the third terms in equation (3.7) are handled in exactly the same way as the corresponding terms of equation (2.20). The sdependent factors of the second term are given by equation (2.25) (where the upper index $R_{i}$ of the third factor is replaced by the lower index $\epsilon_{i}$ ) and the term

$M\left(\epsilon_{i} ;\left(R_{i}^{-1} Q s+R_{i}^{-1} w-m\left(R_{i}^{-1}, R_{i}\right)+R_{i}^{-1} m\left(R, R_{i}\right)+m\left(R_{i}^{-1}, R R_{i}\right)\right), R_{i}^{-1} R R_{i}\right)$.

Again we can write equation (2.26) (without the upper index $R_{i}$ of the last factor of the right-hand side) and for the new term we can write with equation (3.4) of van den 
Broek (1976)

$$
\begin{aligned}
M\left(\epsilon_{i} ;\left(R_{i}^{-1} Q s\right.\right. & \left.\left.+R_{i}^{-1} w-m\left(R_{i}^{-1}, R_{i}\right)+R_{i}^{-1} \boldsymbol{m}\left(R, R_{i}\right)+\boldsymbol{m}\left(R_{i}^{-1}, R R_{i}\right), R_{i}^{-1} R R_{i}\right)\right) \\
= & M\left(\epsilon_{i} ;\left(R_{i}^{-1} Q s, E\right)\right) M\left(\epsilon_{i} ;\left(R_{i}^{-1} w-m\left(R_{i}^{-1}, R_{i}\right)+R_{i}^{-1} \boldsymbol{m}\left(R, R_{i}\right)\right.\right. \\
& \left.\left.+\boldsymbol{m}\left(R_{i}^{-1}, R R_{i}\right), R_{i}^{-1} R R_{i}\right)\right) \delta\left(\epsilon_{i}\right) \gamma^{2}\left(R_{i}^{-1} Q s, R_{i}^{-1} w-m\left(R_{i}^{-1}, R_{i}\right)\right. \\
& \left.+R_{i}^{-1} \boldsymbol{m}\left(R, R_{i}\right)+\boldsymbol{m}\left(R_{i}^{-1}, R R_{i}\right)\right)
\end{aligned}
$$

where $\delta\left(\epsilon_{i}\right)$ is defined by $\delta(1)=0$ and $\delta(-1)=1$.

For $f_{2}(s)$ we obtain an expression analogous to equation (2.30); however, there is now an extra factor $M\left(\epsilon_{i} ;\left(R_{i}^{-1} Q s, E\right)\right)$. If we define $\boldsymbol{k}_{+1}$ to be the zero vector we can write from equations (3.4) and (3.5)

$$
M\left(\epsilon_{i} ;\left(R_{i}^{-1} Q s, E\right)\right)=\exp \left(2 \pi i Q R_{i}^{-1 \mathrm{~T}} \boldsymbol{k}_{\epsilon_{1}}, \boldsymbol{s}\right) .
$$

Therefore the final result is that the matrix elements $\left(\phi_{m}^{o . a}, O_{k}^{\mu} \phi_{l}^{\nu, b}\right)$ are equal to zero for each $m, k$ and $l$ unless there exist values for $i$ and $j$ such that

$\epsilon_{1, i} Q R_{i, i}^{-1 \mathrm{~T}} \boldsymbol{k}^{\prime \prime}-\epsilon_{i} Q R_{i}^{-1 \mathrm{~T}} Q^{-1} \boldsymbol{k}^{\prime}+\boldsymbol{k}-Q \boldsymbol{k}\left(\boldsymbol{R}_{i}\right)+\boldsymbol{p}\left(R_{i}\right)+Q R_{i}^{-1 \mathrm{~T}} \boldsymbol{k}_{\boldsymbol{\epsilon}_{i}}=0 \quad(\bmod 1)$

Now let $\boldsymbol{G}$ be a Shubnikov space group of type IV and $G$ its unitary subgroup. The notation of elements of $G$ will be as before, and elements of $\boldsymbol{G}$ will be denoted by $(t, R, \epsilon)$ where $(t, R) \in G$ and $\epsilon$ equals \pm 1 . The action of $(t, R, \epsilon)$ on space-time is given by

$$
(t, R, \epsilon)(x ; t)=\left(R x+t+t_{R}+\delta(\epsilon) R t_{0}, \epsilon t\right)
$$

where $\delta(\epsilon)$ is defined by $\delta(1)=0$ and $\delta(-1)=1 ; t_{0}$ is a translation which does not belong to the subgroup $H$ of translations of $G$, but $2 t_{0}$ does belong to $H$. The multiplication of elements of $\boldsymbol{G}$ is given by

$(t, R, \epsilon)\left(t^{\prime}, R^{\prime}, \epsilon^{\prime}\right)=\left(t+R t^{\prime}+m\left(R, R^{\prime}\right)+\delta(\epsilon) R t_{0}-\delta(\epsilon) \epsilon^{\prime} R R^{\prime} t_{0}, R R^{\prime}, \epsilon \epsilon^{\prime}\right)$.

Let $\omega$ be a factor system of $\boldsymbol{G}$. From van den Broek $(1977 \mathrm{a}, \mathrm{b})$ it follows that $\omega$ can be chosen from its equivalence class in such a way that

$$
\begin{aligned}
\omega((t, R, \epsilon), & \left.\left(t^{\prime}, R^{\prime}, \epsilon^{\prime}\right)\right) \\
& =\sigma\left((t, R),\left(t^{\prime}, R^{\prime}\right)_{\epsilon}\right) \sigma\left((t, R)\left(t^{\prime}, R^{\prime}\right)_{\epsilon}, n\left(\epsilon, \epsilon^{\prime}\right)\right) N\left(\epsilon, \epsilon^{\prime}\right) M\left(\epsilon ;\left(t^{\prime}, R^{\prime}\right)\right)
\end{aligned}
$$

where $\sigma$ is a factor system of $G$ which has all the properties of the previous section; $n\left(\epsilon, \epsilon^{\prime}\right)$ is given by

$$
n(1,1)=n(1,-1)=n(-1,1)=(e, E)
$$

and

$$
n(-1,-1)=\left(2 t_{0}, E\right) .
$$

$(t, R)_{\epsilon}$ is given by $(t, R)_{1}=(t, R)$ and $(t, R)_{-1}=\left(t+t_{0}-R t_{0}, R\right)$, and $M$ satisfies equations (3.4) and (3.5).

The derivation of the selection rules in this case is analogous to the case of Shubnikov space groups of type II. The only difference is the appearance of the translation $t_{0}$ in the calculations, but in the final result $t_{0}$ cancels, so we obtain again equation (3.10) as a necessary condition of the non-vanishing of matrix elements. 


\section{Acknowledgment}

I thank Dr T Janssen for reading the manuscript.

\section{References}

Bargmann V 1954 Ann. Math., NY 59 1-46

Bradley C J 1966 J. Math. Phys. $71145-52$

Bradley C J and Cracknell A P 1972 The Mathematical Theory of Symmetry in Solids (Oxford: Clarendon Press)

van den Broek P M 1976 J. Phys. A: Math. Gen. $9855-62$

- 1977a J. Phys. A: Math. Gen. $10649-58$

1977b J. Phys. A: Math. Gen. $101653-63$

1978 J. Phys. A: Math. Gen. 11 813-20

Cracknell A P and Davies B L 1976 Group Theoretical Methods in Physics: Lecture Notes in Physics No. 50 eds A Janner, T Janssen and M Boon (Berlin, Heidelberg, New York: Springer-Verlag) pp 338-47

Shaw R and Lever J 1974 Commun. Math. Phys. 38 257-77

Wigner E P 1939 Ann. Math. 40 149-204 